\title{
Las Competencias Ciudadanas en la dimensión Relaciona T del Programa Construye T
}

\section{Citizenship Competencies in the Relaciona T dimension of Construye T Program}

Fecha de recepción:

30 Agosto del 2021

\author{
Mirna Aracely Ruelas Vizcarra ${ }^{1}$ \\ y Edna María Villarreal Peralta ${ }^{2}$
}

Fecha de aprobación: 28 Octubre del 2021

1 Autora de correspondencia. Licenciada en Psicología y Maestra en Innovación Educativa por la Universidad de Sonora. Experiencia docente en preescolar, primaria y secundaria. Actualmente se desempeña como docente en una escuela secundaria.

Correo mirnaruelas94@gmail.com. ORCID: https://oooo-ooo2-2417-06o8

2 Doctora en Economía Aplicada por la Universidad Autónoma de Barcelona y Posgrado en Educación Emocional y Bienestar en la Universidad de Barcelona. Profesora-Investigadora Titular de Tiempo Completo en el Departamento de Economía de la Universidad de Sonora.

Correo: edna.villarreal@unison.mx. ORCID: https://oooo-ooo3-3676-3563

\section{Resumen}

En este trabajo se muestra la importancia de formar ciudadanos, específicamente a la juventud escolarizada de Educación Media Superior en México, para ello se realizó una revisión sistemática del Programa Construye $\mathrm{T}$, el cual incluye una dimensión llamada Relaciona $\mathrm{T}$, enfocada en el desarrollo de las habilidades de conciencia social y colaboración. El objetivo consiste en clasificar las competencias ciudadanas desde una perspectiva teórica en la dimensión mencionada anteriormente. Para ello se utilizaron los pasos propuestos por Torgerson (2003), para una revisión sistemática. Los principales resultados indican que sí es posible clasificar las competencias ciudadanas en las habilidades de conciencia social y colaboración, aun y cuando no están explícitas dichas competencias, pueden ser promovidas a través de las lecciones que ofrece el programa.

Palabras clave: juventud, competencias ciudadanas, programa construye $t$, revisión sistemática.

\section{Abstract}

This paper shows the importance of training citizens, specifically to young people in High School Education in Mexico. For this purpose, a systematic review of the Construye T Program was carried out, which includes a dimension called Relaciona T, focused on skills development of social awareness and collaboration. The objective is to classify citizenship competencies from a theoretical perspective in the aforementioned dimension. For this purpose, the steps proposed by Torgerson (2003, cited in Flores-Crespo) for a systematic review were used. The main results indicate that it was possible to classify citizenship competencies in social awareness and collaboration skills. Even though these competencies are not explicit, they can be promoted through the lessons offered by the program.

Keywords: youths, citizenship competencies, construye T program, systematic review.

\section{FORMACIÓN CIUDADANA}

El desarrollo de ciudadanía en las personas resulta un factor importante para su formación académica, personal y socioemocional, ya que les permite estar informados acerca de los acontecimientos que afectan la sociedad, la economía, el medio ambiente, la educación, entre otros, además se esperaría que adquirieran pensamiento crítico al momento de analizar y tomar decisiones que afecten a ellos y a los demás. La formación ciudadana en la juventud 
específicamente resulta decisiva para su desarrollo personal y el impacto social que esta pueda tener. Se estima que la juventud es el grupo poblacional más interconectado globalmente, lo que permite una interacción inmediata con lo que sucede en su lugar de residencia y en otras partes del mundo. Así mismo, son beneficiados por organismos internacionales que proponen programas y proyectos donde se les incluye como actores principales, de una manera especial y no más importante, son los jóvenes escolarizados, ya que estos tienen la posibilidad de experimentar estos programas y desarrollarlos.

\section{1 ¿Desde cuándo se han venido formando ciudadanos?}

La ciudadanía cobra cada vez mayor relevancia debido a los cambios que se producen en los sistemas mundiales, hace más de 100 años se comienza a consolidar universalmente algunos beneficios para las personas, los que pertenecían a clases altas fueron los primeros en recibirlos, después y con mucho trabajo, esos beneficios llegan poco a poco a los más marginados.

Los modelos de ciudadanía aportan elementos al desarrollo de los individuos, permiten y garantizan ciertos beneficios. Algunos modelos de ciudadanía contemporáneos son: ciudadanía liberal: se caracteriza por defender la libertad, el uso instrumental de la moral pública y el individualismo; ciudadanía republicana: se enfoca en corregir las desigualdades y tiene la idea de que el ciudadano no nace, sino que "se hace", apuesta por la educación de este; ciudadanía comunitarista: la comunidad privilegia al individuo; ciudadanía multicultural: pone énfasis en grupos sociales desfavorecidos como mujeres, migrantes o minorías (Horrach, 2009). El modelo de ciudadanía de Marshall (1949) promueve la igualdad y resalta los derechos de los ciudadanos, agrupándolos comúnmente en los siguientes:

- Derechos civiles: consiste en los derechos necesarios para la libertad individual: libertad de la persona, libertad de expresión, de pensamiento y de religión, el derecho a la propiedad, a cerrar contratos válidos y el derecho a la justicia (derecho de defender y hacer valer todos los derechos de uno en términos de igualdad con otros y mediante los procedimientos legales) Instituciones asociadas a los tribunales.
- Derechos políticos: garantizan el derecho a participar del poder político como miembro de un cuerpo investido de autoridad política, o como elector de los miembros de tal cuerpo. Instituciones del parlamento y consejos del gobierno.

- Derechos sociales: todo ciudadano tiene desde el derecho a un mínimo de bienestar económico y seguridad al derecho a participary a vivir la vida de un ser civilizado conforme a los estándares corrientes en la sociedad. Sistema educativo y servicios sociales.

Además de lo anterior, postula que existe un tipo de igualdad básica asociada al concepto de la pertenencia plena a una comunidad (o a la ciudadanía), algo que no es inconsistente con las desigualdades que diferencian los distintos niveles económicos en la sociedad.

La finalidad de formar ciudadanos en el mundo aspira a la democracia, según Sartori (2012) "el pueblo que decide en términos de principio mayoritario absoluto es, la mayoría de las veces, un cuerpo que representa al pueblo y que refleja, generalmente, a la mayoría popular que lo elije. $\mathrm{Al}$ final de este trayecto de todas formas sigue siendo válido que el pueblo contabilizado por el principio mayoritario absoluto se divide en una mayoría que se lleva todo y una minoría que lo pierde todo. Lo cual permite a la mayoría si así lo desea, reducir a la minoría o a la impotencia. Lo que no puede permitirse" (p. 9).

Sin embargo, tratar de lograrlo a un nivel global o nacional con cada ciudadano puede resultar complejo, es por lo que en este trabajo se pone énfasis en jóvenes escolarizados, específicamente en Educación Media Superior, Bolívar (2016) "una educación democrática, en el doble sentido de educar para la democracia y en la democracia, es a la vez un fin y un medio de la educación. En una sociedad democrática constituye una obligación de la educación pública capacitar a los futuros ciudadanos para poder participar activamente en la sociedad civil y política sin riesgo de exclusión, lo que supone asegurar que toda la población adquiera aquellas virtudes, conocimientos y habilidades necesarios para la participación políticay la inserción social” (p. 71). Así mismo señala que la formación ciudadana no sólo debe limitarse a una asignatura que se enseña, sino como una tarea de toda la institución educativa. También menciona que los 
procesos del aula, el clima del centro educativo y el servicio a la comunidad son importantes para la formación ciudadana, por ejemplo: enseñar los valores tales como honestidad, responsabilidad, libertad entre otros, así como promover procesos de participación: reflexiones críticas, debates, aprendizaje cooperativo, entre otros. La escuela debe organizarse democráticamente, esto lleva a construir un sentido de pertenencia y el empleo de metodologías que lleven a la puesta en práctica de los valores democráticos y el aprendizaje-servicio como metodología idónea para la práctica ciudadana, en esta, se combina la participación con el servicio a la comunidad y la enseñanza de conocimientos y valores.

Tabla 1. Programas de ciudadanía

\subsection{Programas de formación ciudadana}

Para dar un vistazo actual de la formación ciudadana a nivel mundial, se muestran en la Tabla 1 algunos programas aplicados en distintos países, los cuales, fueron publicados por el Banco Interamericano de Desarrollo (2019). Estos programas, aunque se clasifican en el tema de ciudadanía también se valen de temas como habilidades emocionales, empleo en jóvenes, combate a la violencia, género, medio ambiente, entre otros.

\subsection{El Programa Construye T}

En México, el programa Construye T se enfoca en el desarrollo de habilidades socioemocionales, pero a su vez desarrolla otras áreas como la ciudadana,

\begin{tabular}{|c|c|}
\hline Programa & Objetivo \\
\hline $\begin{array}{l}\text { Programa de } \\
\text { ciudadanía } \\
\text { y liderazgo } \\
\text { (Paraguay) }\end{array}$ & $\begin{array}{l}\text { El programa se desarrolla en tres etapas: en la primera, los jóvenes realizan una identificación de los problemas que hay } \\
\text { en su comunidad. Después, por siete días detectan las soluciones para resolver los problemas, el trabajo de campo es muy } \\
\text { importante en esta etapa. Aquí los participantes se reconectan con el entorno que los rodea, con su familia, su escuela y su } \\
\text { comunidad. En la segunda fase se les capacita en arte, deporte, tecnología, entre otras. Y en la tercera fase vuelcan lo que } \\
\text { han aprendido en su escuela, apoyando a los docentes con los niños y las niñas de primaria, para que estos puedan tener } \\
\text { programas de deporte, arte, programación, etc. Desarrollado por la Fundación Scholas Ocurrentes de Paraguay (citado } \\
\text { por Mateo, 2019, p.18o). Competencia: Ciudadanía para desarrollar liderazgo. }\end{array}$ \\
\hline $\begin{array}{l}\text { TransFórmate } \\
\text { (Colombia) }\end{array}$ & $\begin{array}{l}\text { Ofrece a jóvenes víctimas del conflicto armado (de entre } 16 \text { y } 29 \text { años) la posibilidad de fortalecer sus capacidades para } \\
\text { facilitar su participación en actividades sociales, productivas y de generación de ingreso. Lo hace a través de un módulo } \\
\text { de formación en habilidades socioemocionales y un curso de formación en habilidades técnicas conducentes a un título } \\
\text { técnico laboral que viene a durar entre } 12 \text { y } 18 \text { meses. Desarrollado por el Ministerio de Trabajo colombiano (citado por } \\
\text { Mateo, 2019, p.181). Competencia: Ciudadanía para jóvenes víctimas de violencia. }\end{array}$ \\
\hline $\begin{array}{l}\text { Sácale tarjeta roja a } \\
\text { la violencia (Perú) }\end{array}$ & $\begin{array}{l}\text { El objetivo consistía en animar a los estudiantes a plantarle cara al bullying, a prestar ayuda a sus víctimas y a denunciar } \\
\text { los actos violentos de los que sean testigos. Eran talleres teóricos sobre las consecuencias del acoso, actividades prácticas } \\
\text { para reforzar los mensajes del taller y distribución de material informativo. Además, se desarrolló una plataforma digital } \\
\text { para que los estudiantes, padres y amigos puedan denunciar de manera anónima (Gutiérrez, et al., 2018, citado en Mateo, } \\
\text { 2019, p.182). Dimensión: ciudadanía para jóvenes víctimas de violencia. Competencia: ciudadanos contra el acoso escolar. }\end{array}$ \\
\hline $\begin{array}{l}\text { Aulas para la paz } \\
\text { (Colombia) }\end{array}$ & $\begin{array}{l}\text { Este programa que combinaba varios componentes. Por un lado, incluía un componente en el aula, para los grados de } \\
\text { segundo a quinto de primaria, basado en el desarrollo de competencias cívicas en temas como la agresión, el conflicto o la } \\
\text { intimidación, y la empatía, el manejo constructivo de la rabia y asertividad. También incluía un componente familiar, que } \\
\text { consistía en visitas familiares, buscando que los padres desarrollaran las mismas competencias que los niños. Por último, } \\
\text { un componente de grupos heterogéneos conformados por dos estudiantes con altos niveles de agresión y cuatro destaca- } \\
\text { dos por sus comportamientos prosociales (Chaux, et al., 2017, citado por Mateo, 2019, p.183). Competencia: ciudadanos } \\
\text { contra el acoso escolar. }\end{array}$ \\
\hline $\begin{array}{l}\text { Súbete } \\
\text { (Uruguay) }\end{array}$ & $\begin{array}{l}\text { Es un programa de educación medioambiental que pretende animar a la niñez y la juventud a utilizar su creatividad y su } \\
\text { energía para participar activamente en proyectos que promuevan y mejoren la sostenibilidad en sus propias comunidades } \\
\text { (citado por Mateo, 2019, p.183). Dimensión: ciudadanía para el medio ambiente. Competencia: ciudadanía para el medio } \\
\text { ambiente. }\end{array}$ \\
\hline $\begin{array}{l}\text { Amor del bueno } \\
\text { (México) }\end{array}$ & $\begin{array}{l}\text { Tiene como objetivo que la comunidad escolar asuma una postura crítica hacia la violencia de género promoviendo una } \\
\text { convivencia incluyente, igualitaria y no violenta entre los jóvenes. Consta de dos componentes: talleres con los estudian- } \\
\text { tes y mejora del ambiente escolar (Sosa Rubi, et al., 2016, citado en Mateo, 2019, p.184). Competencia: ciudadanía por la } \\
\text { igualdad de género. }\end{array}$ \\
\hline
\end{tabular}

Fuente: elaboración propia con datos del Banco Interamericano de Desarrollo (2019). 
este programa comenzó en 2008 bajo el nombre de Programa de Prevención de Riesgos en la Educación Media Superior (PPREMS) su objetivo principal era combatir el abandono escolar. Sin embargo, un año después cambió su nombre a Construye T y se unió el Programa de las Naciones Unidas para el Desarrollo (PNUD) para dar apoyo logístico, económico, organizacional, etc., el objetivo seguía siendo el mismo, fue hasta 2014 que el programa tuvo un cambio favorable, ya que se integraron las habilidades socioemocionales, cuyo objetivo consiste en fortalecer las capacidades de la escuela para desarrollar habilidades socioemocionales en las y los estudiantes y así mejorar el ambiente escolar en los planteles públicos de educación media superior que participan en el programa (PNUD 2019). En la tabla 2 se puede observar el marco conceptual de Construye T, la dimensión abarca dos habilidades generales, las cuales a su vez integran habilidades específicas.

Tabla 2. Marco conceptual de Construye T

\begin{tabular}{|c|c|c|}
\hline Dimensión & Habilidades & Habilidades específicas \\
\hline \multirow{3}{*}{ Conoce $\mathrm{T}$} & Autoconciencia & $\begin{array}{l}\text { 1. Autopercepción } \\
\text { 2. Autoeficacia } \\
\text { 3. Reconocimiento de } \\
\text { emociones }\end{array}$ \\
\hline & Autorregulación & $\begin{array}{l}\text { 4. Manejo de emociones } \\
\text { 5. Postergación de la } \\
\text { gratificación } \\
\text { 6. Tolerancia a la } \\
\text { frustración }\end{array}$ \\
\hline & Determinación & $\begin{array}{l}\text { 7. Motivación al logro } \\
\text { 8. Perseverancia } \\
\text { 9. Manejo del estrés }\end{array}$ \\
\hline \multirow[b]{2}{*}{ Relaciona T } & Conciencia social & $\begin{array}{l}\text { 10. Empatía } \\
\text { 11. Escucha activa } \\
\text { 12. Toma de perspectiva }\end{array}$ \\
\hline & $\begin{array}{l}\text { Relación con los } \\
\text { demás }\end{array}$ & $\begin{array}{l}\text { 13. Asertividad } \\
\text { 14. Manejo de conflictos } \\
\text { interpersonales } \\
\text { 15. Comportamiento } \\
\text { prosocial }\end{array}$ \\
\hline Elige T & $\begin{array}{c}\text { Toma responsable de } \\
\text { decisiones }\end{array}$ & $\begin{array}{l}\text { 16. Generación de opciones } \\
\text { y consideración de } \\
\text { consecuencias } \\
\text { 17. Pensamiento crítico } \\
\text { 18. Análisis de } \\
\text { consecuencias }\end{array}$ \\
\hline
\end{tabular}

Fuente: elaboración propia con datos del PNUD (2019).
Las habilidades generales que se encuentran en la tabla anterior se distribuyen durante los tres años que dura la formación en Educación Media Superior, se adiciona una habilidad más en la dimensión Elige T que se llama Perseverancia, la cual incluye temas sobre proyecto de vida. En la figura 1 se muestran las habilidades distribuidas por semestre, cada una con 12 lecciones para ser impartidas en clase.

La figura anterior muestra las seis habilidades que se manejan en los 6 semestres que dura el bachillerato. Como se puede apreciar, cada habilidad tiene 12 lecciones, las cuales, a su vez tienen 6 sesiones, esto se contempla para que cada día se trabaje una sesión con una duración de 20 minutos. Para este trabajo solo se tomaron las lecciones de conciencia social y colaboración que corresponden a la dimensión de Relaciona T. En el apartado de resultados se mostrará las sesiones en las que se clasificaron las competencias ciudadanas.

Hasta 2018 el Programa Construye T se aplicó en 9,729 planteles públicos federales y estatales de Educación Media (PNUD, 2015). Como se mencionó anteriormente, este programa no trata directamente el tema de ciudadanía, su principal enfoque está dirigido al desarrollo de Habilidades Socioemocionales, sin embargo, se pretende encontrar los elementos de ciudadanía que este incluye, información que incluso se encuentra implícita en el programa.

Por lo tanto el objetivo de esta investigación consiste en clasificar las competencias ciudadanas que contiene el Programa Construye T en la dimensión de Relaciona T, específicamente en las lecciones de las habilidades de conciencia social y colaboración.

\section{COMPETENCIAS CIUDADANAS}

Para la identificación de competencias ciudadanas se retomó la perspectiva de Chaux, Lleras y Velásquez (2004) quienes proponen una formación ciudadana, que se basa en cinco principios: 1) abarcar todas las competencias necesarias para la acción ciudadana; 2) brindar múltiples oportunidades para la práctica de las competencias; 3 ) integrar la formación ciudadana de manera transversal en las áreas académicas; 4) involucrar a toda la comunidad educativa; 5) evaluar el impacto. 


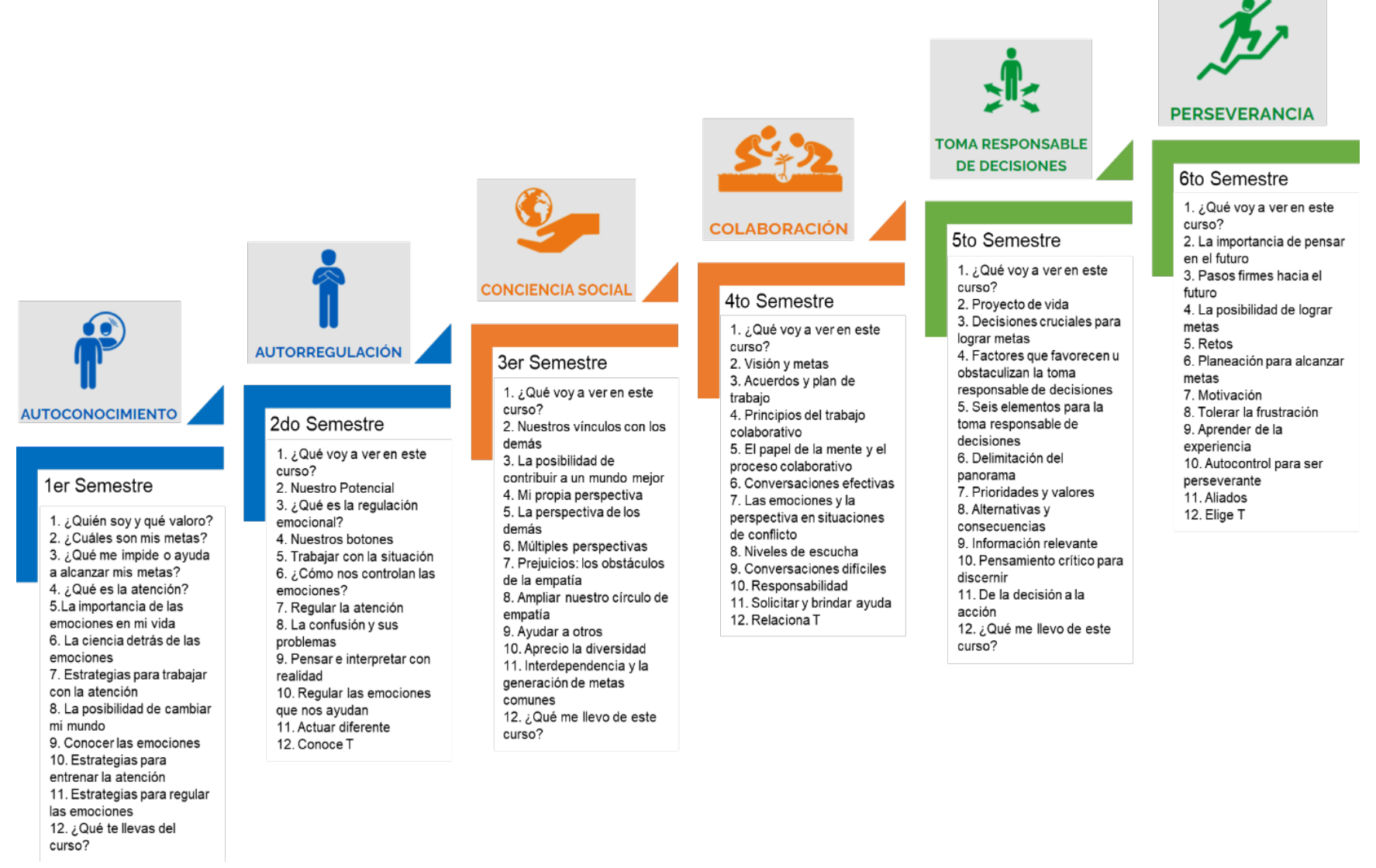

Figura 1. Lecciones por semestre en el Programa Construye T

Fuente: elaboración propia con datos de Construye T (2021)

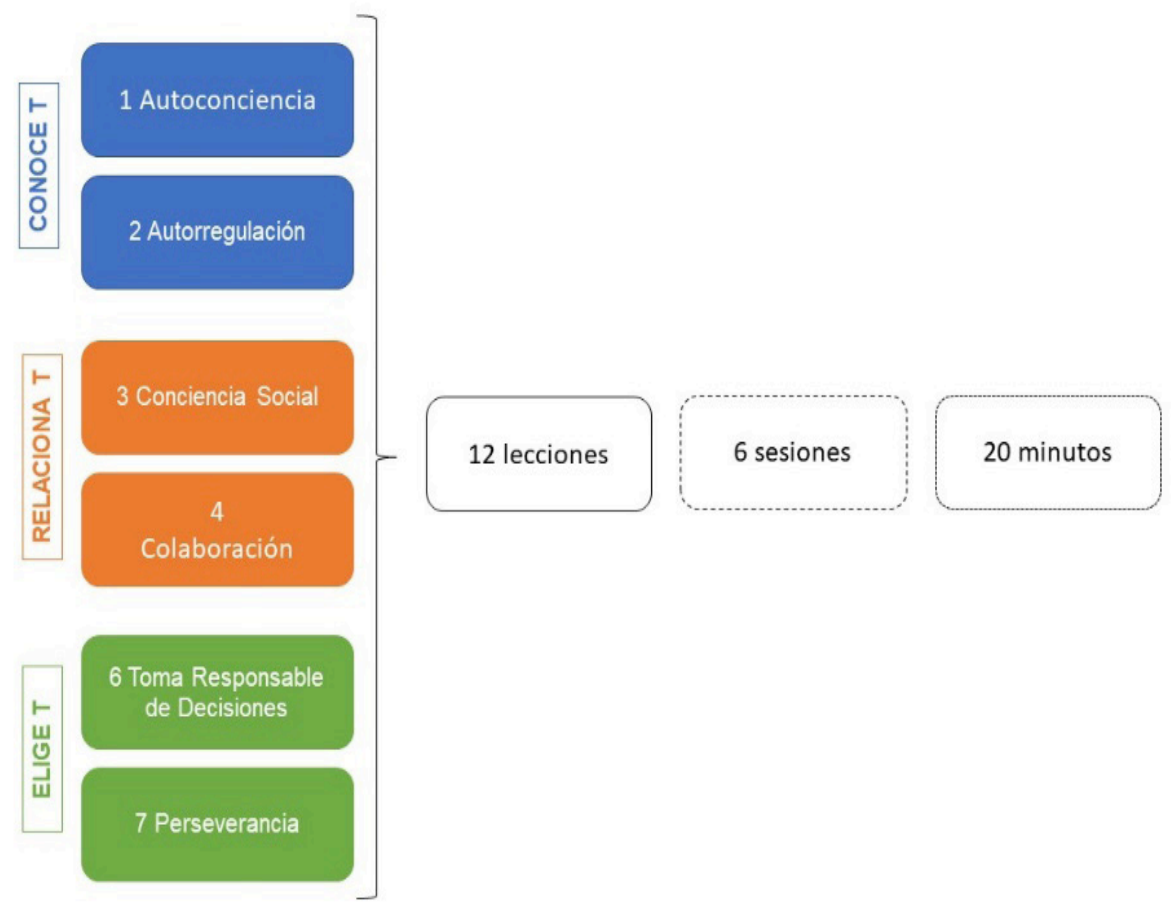

Figura 2. Distribución del Programa Construye T

Fuente: elaboración propia con datos de Construye T (s.f.) 
Ruiz y Chaux (2005) definen competencias ciudadanas como el "conjunto de capacidades y habilidades cognitivas, emocionales y comunicativas -integradas- relacionadas con conocimientos básicos (contenidos, procedimientos, mecanismos) que orientan moral y políticamente nuestra acción ciudadana (p.32)". Es importante resaltar que la ciudadanía debe ejercerse de manera autónoma y no impostada por otros, este sería el objetivo fundamental de la formación ciudadana. A continuación, se definen los componentes de las competencias ciudadanas.

- Conocimientos (CO): se refiere a la "comprensión de información, procedimientos, estrategias y contenidos que necesitan las personas para el ejercicio de la ciudadanía" (Ruiz y Chaux, 2005, p. 44). La propuesta que hace el autor sobre conocimientos que debieran aprender en la escuela son: los derechos fundamentales, la Constitución Política, mecanismos, procedimientos e instancias de participación democrática, estrategias y mecanismos para la resolución de conflictos, entre otros.

- Competencias cognitivas (CCG): es la "capacidad de realizar diversos procesos mentales, en este caso, para ejercer la ciudadanía". Chaux, Lleras y Velásquez (2004, p. 21 y 22) proponen algunas competencias cognitivas:

- Toma de perspectiva: lograr acuerdos de beneficio común con los demás.

- Interpretación de intenciones: evaluar de manera adecuada las intenciones y propósitos de las acciones de los demás.

- Generación de opciones: imaginarse muchas maneras de resolver un conflicto.

- Consideración de consecuencias: efectos que puede tener las alternativas de solución.

- Metacognición: capacidad de reflexionar sobre sí mismo.

- Pensamiento crítico: cuestionar y evaluar la validez de cualquier creencia.

- Competencias emocionales (CE): "capacidades necesarias para identificar las emociones propias y las de los otros y responder a ellas de forma constructiva" (Ruiz y Chaux, 2005, p.40)". Las siguientes competencias emocionales son propuestas por Chaux, Lleras y Velásquez (2004, p. 23).
- Identificación de las propias emociones: reconocer y nombrar las emociones.

- Manejo de las propias emociones: capacidad de dominar las emociones.

- Empatía: capacidad de sentir o comprender lo que otros sienten.

- Identificación de las emociones de los demás: identificar lo que pueden estar sintiendo otras personas.

- Competencias comunicativas (CCM): "habilidades que permiten entablar diálogos constructivos con los demás, comunicar nuestros puntos de vista, posiciones, necesidades, intereses e ideas, en general, y comprender aquellos que los demás ciudadanos buscan comunicar" (Chaux, Lleras y Velásquez, p. 24). Así mismo, estos autores proponen las siguientes competencias comunicativas:

- Escucha activa: estar atento a lo que dicen los demás y demostrar que se está escuchando.

- Asertividad: consiste en expresar las necesidades, intereses, posiciones, derechos e ideas de manera clara.

- Argumentación: capacidad de expresar y sustentar una posición.

- Competencias integradoras (CI): "son aquellas competencias más amplias y abarcadoras que, en la práctica, articulan los conocimientos y las competencias cognitivas, emocionales o comunicativas" (Chaux, Lleras y Velásquez, 2004, p. 24).

Según Ruiz y Chaux (2005) el ejercicio de la ciudadanía en y desde la escuela lleva consigo tres retos: 1) convivir pacífica y contractivamente con otros, 2) construir acuerdos y normas de manera colectiva para el bien común, y 3 ) construir sociedad a partir de las diferencias y no a pesar de ellas.

Si bien el Programa Construye T no tiene integrado un marco de referencia sobre formación ciudadana, su marco de referencia

\section{METODOLOGÍA}

\subsection{Metodología}

Para cumplir con el objetivo de este trabajo de investigación se desarrolla una metodología amplia, basada en la revisión sistemática de la información existente del Programa Construye T. 
Según Sánchez-Meca (2010) la revisión sistemática "es un tipo de investigación científica mediante la cual se revisa la literatura científica sobre un tópico partiendo de una pregunta formulada de forma clara y objetiva, utilizando métodos sistemáticos y explícitos para localizar, seleccionar y valorar críticamente las investigaciones relevantes a dicha pregunta y aplicando protocolos sistemáticos para la recogida de datos e información de dichas investigaciones, con el fin de alcanzar conclusiones válidas y objetivas sobre qué es lo que dicen las evidencias sobre dicho tópico" (p.54).

Para llevar a cabo la revisión sistemática se siguieron una serie de pasos propuestos por Torgerson (2003), que consisten en: 1) diseñar un protocolo con la finalidad de establecer las bases teóricas, empíricas y conceptuales del tema. Además de establecer la pregunta de investigación, los objetivos, los métodos de búsqueda, validación, resumen, calidad y síntesis; 2) definir los criterios para la inclusión o exclusión de materiales en donde se debe especificar si los estudios elegidos serán solamente experimentales y escritos en un idioma determinado; 3 ) el protocolo debe ser revisado por los pares para así comenzar la búsqueda de literatura en revistas especializadas clave; 4) mapeo, en donde se describen y clasifican los estudios hallados en la primera revisión. Aquí es donde se refina la búsqueda del material bibliográfico que será definitivo para la elaboración de la revisión sistemática; 5) una vez que el material se ha identificado, se debe extraer la información a partir de una hoja de datos; 6) después de extraer la información de los estudios seleccionados, se realiza una "síntesis", puede hacerse de manera cualitativa si los datos no permiten una síntesis numérica, 7) la fase final de la elaboración de la revisión sistemática incluye la interpretación de la información sintetizada.

\section{RESULTADOS}

A lo largo de la investigación se fueron cumpliendo los pasos de la revisión sistemática, sin embargo, en este trabajo solo se muestra el paso siete de la revisión sistemática, que consiste en interpretar la información sintetizada, así entonces, se presenta a continuación la identificación de las competencias ciudadanas en la dimensión de Relaciona $\mathrm{T}$ : conciencia socialy colaboración. Para ello se tomaron como referencia las competencias ciudadanas propuesta por Chaux (2004): 1) conocimientos (CO), 2) competencias cognitivas (CCG), 3) competencias emocionales (CE), 4) competencias comunicativas $(\mathrm{CCM}), 5)$ competencias integradoras $(\mathrm{CI})$.

Como se mencionó en la figura 2 cada habilidad socioemocional (conciencia social y colaboración) contiene 12 lecciones, y cada una de estas incluye seis sesiones. Para realizar la identificación se seleccionó una sesión aleatoria de cada lección y se comparó su objetivo y contenidos con la definición alguna de las competencias ciudadanas propuestas por Chaux (2004). En la tabla 3 se observan cuáles son las competencias ciudadanas que se encuentran en las sesiones tanto de conciencia social como colaboración.

Como se puede observar en la tabla 3, las sesiones seleccionadas pudieron ser identificadas en las competencias ciudadanas, excepto por la lección 12 de conciencia social y las lecciones 10 y 12 de colaboración. La competencia ciudadana que más se encontró en las sesiones fue la de conocimiento (CO), se esperaría que los jóvenes pudieran desarrollar mayor conocimiento de ellos mismos, de sus derechos, obligaciones, valores humanos, pero también para resolver conflictos o solucionar problemas. Las competencias comunicativas (CCG) se encontraron en 3 sesiones, esto permitiría a los estudiantes que puedan tomar perspectiva, interpretarintenciones, generaropciones, considerar consecuencias y desarrollar metacognición según (Chaux, Lleras y Velásquez, 2004). En cuanto a las competencias emocionales (CE) se encontraron en tres sesiones, los estudiantes podrán además de autoconocer sus emociones, conocer o identificar la de los demás, desarrollar empatía, manejar sus emociones y por ende manejar situaciones, esto desde la perspectiva de (Chaux, Lleras y Velásquez, 2004).

Así mismo, las competencias comunicativas (CCM) fueron encontradas en tres sesiones, lo cual llevaría a los jóvenes a que puedan tener la capacidad de argumentar sus ideas, comunicarse asertivamente, pero también escuchar a los otros. Por otra parte, las competencias integradoras (CI) fueron identificadas en cuatro sesiones, según (Chaux, Lleras y Velásquez, 2004) estas competencias integran tanto los conocimientos, como las comunicativas, cognitivas y emocionales, así entonces se infiere que las sesiones en las que se encontraron ofrecen al estudiante una formación más integral. 
Tabla 3. Competencias ciudadanas que se encuentran en las lecciones de Relaciona T

\begin{tabular}{|c|c|c|}
\hline Lecciones & $\begin{array}{c}\text { Conciencia social (3er semestre) } \\
\text { Sesiones }\end{array}$ & $\begin{array}{l}\text { Competencias } \\
\text { ciudadanas }\end{array}$ \\
\hline 1. ¿Qué voy a ver en este curso? & 1.5 Somos comunidad & CCG \\
\hline 2. Nuestros vínculos con los demás & 2.4 Convivir desde la empatía & $\mathrm{CE}$ \\
\hline 3. La posibilidad de contribuir a un mundo mejor & 3.6 Plan para propiciar relaciones constructivas & $\mathrm{CI}$ \\
\hline 4. Mi propia perspectiva & $4 \cdot 3$ ¿Y la perspectiva del otro? & $\mathrm{CCM}$ \\
\hline 5. La perspectiva de los demás & 5.5 Amar es: hablar y escuchar & $\mathrm{CCM}$ \\
\hline 6. Múltiples perspectivas & 6.3 Mi perspectiva y la de los demás & $\mathrm{CI}$ \\
\hline 7. Prejuicios: obstáculos a la empatía & $7 \cdot 5$ ¿Tengo prejuicios? & $\mathrm{CO}$ \\
\hline 8. Ampliar nuestro círculo de empatía & 8.5 La mirada de los otros & $\mathrm{CE}$ \\
\hline 9. Ayudar a otros & 9.2 Si te caes, te levanto & CCG \\
\hline 10. Aprecio a la diversidad & 10.3 Promotores de la inclusión & $\mathrm{CO}$ \\
\hline $\begin{array}{l}\text { 11. Interdependencia y la generación de metas } \\
\text { comunes }\end{array}$ & 11.3 Solucionamos problemas en comunidad & $\mathrm{CO}$ \\
\hline 12. ¿Qué me llevo de este curso? & - & - \\
\hline Lecciones & $\begin{array}{c}\text { Colaboración (4to semestre) } \\
\text { Sesiones }\end{array}$ & $\begin{array}{l}\text { Competencias } \\
\text { ciudadanas }\end{array}$ \\
\hline 1. ¿Qué voy a ver en este curso? & 1.3 Colaborar en la escuela & $\mathrm{CO}$ \\
\hline 2. Visión y metas & $\begin{array}{l}\text { 2.6 Elaboremos juntos un plan de trabajo } \\
\text { colectivo }\end{array}$ & $\mathrm{CI}$ \\
\hline 3. Acuerdos y plan de trabajo & 3.5 Vamos a tomar acuerdos & $\mathrm{CI}$ \\
\hline 4. Principios del trabajo colaborativo & 4.4 Responsabilidades y compromisos & $\mathrm{CO}$ \\
\hline $\begin{array}{l}\text { 5. El papel de la mente y las emociones en el } \\
\text { proceso colaborativo }\end{array}$ & 5.2 Mi actitud impacta & $\mathrm{CE}$ \\
\hline 6. Conversaciones efectivas & 6.1 Propuesta de solución & $\mathrm{CCM}$ \\
\hline $\begin{array}{l}\text { 7. Las emociones y la perspectiva en situaciones } \\
\text { de conflicto }\end{array}$ & 7.4 Pensar distinto & CCG \\
\hline 8. Niveles de escucha & 8.6 Oídos a la obra & $\mathrm{CCM}$ \\
\hline 9. Conversaciones difíciles & 9.6 La construcción de acuerdos & CCG \\
\hline 10. Responsabilidad & - & - \\
\hline 11. Solicitar y brindar ayuda & 11.3 Ayudar me ayuda & CCG \\
\hline 12. Relaciona T & - & - \\
\hline
\end{tabular}

Fuente: elaboración propia.

Construye T tiene un enfoque en HSE, sin embargo, logra alcanzar otros aspectos como las competencias ciudadanas, este programa tiene un gran alcance en cuanto a planteles, estudiantes, docentes directivos, entre otros, es por ello por lo que se buscó en este y no en otros programas, los elementos de formación ciudadana que pudiera incluir. Se desconoce si los actores involucrados en el programa desarrollan realmente en su vida cotidiana tanto las HSE como las CC, este representaría un campo de investigación importante para darle seguimiento a los resultados del programa.

\section{CONCLUSIONES}

Para la identificación de las competencias ciudadanas se eligió el Programa Construye T porque este representa para la Educación Media Superior y para México una de las intervenciones más completas que se pueden aplicar en estudiantes jóvenes. El objetivo de clasificar las habilidades socioemocionales en las competencias ciudadanas desde la perspectiva de Chaux (2004) se cumplió, lo cual, indica que los estudiantes pueden desarrollar las competencias comunicativas, cognitivas y emocionales al momento de resolver las actividades Construye T, específi- 
camente en la dimensión de Relaciona T. Por otra parte, para precisar que los jóvenes apliquen estas competencias en la vida diaria, serían necesarias otras investigaciones.

La relevancia de esto radica en que tanto el marco de referencia de Construye $\mathrm{T}$ y el de las Competencias Ciudadanas es distinto, pero busca el desarrollo integral de los jóvenes, por eso se dice que implícitamente se trabajan las CC en el programa. Hasta el momento no se encontraron estudios similares que trataran de comparar, clasificar o identificar si algún programa de educación emocional incluyera aspectos de formación ciudadana.

Es muy importante dejar claro que el hecho de haber realizado una clasificación de competencias ciudadanas en el Programa Construye $\mathrm{T} y$ argumentar que éste las integra en sus lecciones, no quiere decir que se lleven a la práctica. Cuando se habla de competencias, se da por hecho que los involucrados tendrán que ejecutarlas, en este caso las competencias sólo son identificadas en la estructura del programa. No se cuenta con la evidencia necesaria para afirmar que los jóvenes estudiantes de México aplican en su vida diaria las CC y las HSE, lo que sí se puede confirmar es que, por lo menos, lo jóvenes estudiantes de México tienen un Programa que los conduce y guía a desarrollar tales competencias y habilidades para que las lleven a la práctica.

Para el desarrollo de competencias ciudadanas el Programa Construye T publicó en 2020 una nueva guía de implementación sobre Práctica y Colaboración Ciudadana, la cual se dirige hacia la formación ciudadana y socioemocional para el desarrollo sostenible, la relación que describen entre HSE y ciudadanía tiene qué ver con que una formación socioemocional es un factor clave para la práctica y colaboración ciudadana, por ejemplo, se puede promover el respeto, el reconocimiento y la valoración a la diversidad a través de las habilidades de conciencia social y colaboración. Así mismo, la conciencia social se puede desarrollar a través de la empatía para comprender a las otras personas, la colaboración potencia la comunicación asertiva y el trabajo colaborativo.

La guía propone una metodología para desarrollar proyectos que consiste en un diagnóstico participativo, una planificación, un seguimiento y una evaluación del proyecto (Construye T, 2020). Esta guía está contemplando la Agenda 2030 y los
Objetivosdel DesarrolloSostenible, porlotanto, trata de integrar elementos del contexto internacional y acercarlos a la educación. Hasta el momento se desconocen los proyectos que se puedan generar de aquí, ya que es una guía relativamente nueva y ha salido en tiempos de pandemia por COVID-19, sería interesante conocer y participar de los proyectos futuros que puedan surgir.

En síntesis, el Programa Construye $\mathrm{T}$, tiene una gran variedad de recursos para directivos, docentes, alumnos, padres de familia, comunidad escolar, entre otros. Hasta ahora el desarrollo de habilidades socioemocionales representa su principal objetivo, para ello se han ido añadiendo contenidos innovadores que responden a las necesidades actuales de los jóvenes de México. Cabe desatacar que sería importante aprovecharse de la promoción y cobertura que este tiene para llevarse a todos los planteles públicos y ofrecerse a los planteles privados, ya que todos tienen el derecho de gozar de una educación socioemocional. En lo que respecta a las competencias ciudadanas, sería conveniente que se de seguimiento a las actividades propuestas para su desarrollo, quizá el incorporar cuestiones más precisas como ambientes democráticos, participación ciudadana, conocimiento de algunos derechos y obligaciones, equiparía aún más a los estudiantes jóvenes de México para su desenvolvimiento en la escuela y en los entornos que lo rodean.

\section{REFERENCIAS}

Banco Interamericano de Desarrollo (2019) El futuro ya está aquí. Habilidades transversales en América Latina y el Caribe en el siglo XXI. http://publications. iadb.org/publications/spanish/document/El-futuroya-est $\% C_{3} \% A_{1}$-aqui-Habilidades-transversales-deAmerica-Latina-y-el-Caribe-en-el-siglo-XXI.pdf

Bolívar, A. (2016). Educar democráticamente para una Ciudadanía Activa. Revista Internacional de Educación para la Justicia Social. 5 (1), p.p. 69-87. Recuperado de: https://revistas.uam.es/riejs/article/ viewFile/4344/4717

Chaux, E. (2005). Estándares Básicos de Competencias Ciudadanas. Formar para la ciudadanía... ¡sí es posible! Recuperado de: https://www.mineducacion. gov.co/1759/articles-116042_archivo_pdf4.pdf

Chaux, Lleras y Velásquez (2004) Competencias ciudadanas: de los estándares al aula. Una propuesta de integración a las áreas académicas. Ministerio de educación, Colombia. 
Construye T (2020) Guía de Práctica y Colaboración Ciudadana para planteles de Educación Media Superior. PNUD. http://construyet.sep.gob.mx/docs/ guia-practica-colaboracion-ciudadana.pdf? $v=1$

Construye $\mathrm{T}$ (2021) Materiales para el aula: modelo ampliado. http://construyet.sep.gob.mx/materialespara-el-aula/2/modelo-ampliado

Construye $\mathrm{T}$ (s.f.) Consideraciones y sugerencias de contenido del Programa Construye T 2021-1. http:// construyet.sep.gob.mx/resources/pdf/guia-rapida/ Documento_informativo_Construye_T.pdf?v=131217

Horrach, J. (2009). Sobre el concepto de ciudadanía: historia y modelos. Factótum, p. 1-22. http:// www.revistafactotum.com/revista/f_6/articulos/ Factotum_6_1_JA_Horrach.pdf

Marshall, T. (1949) Ciudadanía y clase social. Revista Española de Investigaciones Sociológicas. 79. Pp. 297-344. Disponible en: http://www.reis.cis.es/REIS/ PDF/REIS_079_13.pdf

Mateo, M. (2019) Programas de ciudadanía. En Banco Interamericano de Desarrollo. El futuro ya está aquí. Habilidades transversales en América Latina y el
Caribe en el siglo XXI. 162-192. https://publications. iadb.org/publications/spanish/document/El-futuroya-est $\% \mathrm{C}_{3} \% \mathrm{~A}_{1}$-aqui-Habilidades-transversales-deAmerica-Latina-y-el-Caribe-en-el-siglo-XXI.pdf

PNUD (2015) Construye T. https://www.mx.undp.org/ content/mexico/es/home/projects/contruye-t.html

PNUD (2019) Descripción y resultados del Programa Construye T hasta 2019 https://info.undp.org/docs/ pdc/Documents/MEX/PRODOC\%202019_2020.pdf

Ruiz, A., y Chaux, E. (2005). La Formación de Competencias Ciudadanas. Bogotá: ASCOFADE. Recuperado de: https://laasociacion.files.wordpress. com/2015/11/la-formacion-de-competenciasciudadanas.pdf

Sánchez, M. (2010) Cómo realizar una revisión sistemática y un meta-análisis. Aula Abierta. 38 (2) pp. 53-64. https://dialnet.unirioja.es/servlet/ articulo? codigo $=3316651$

Sartori, G. (2012). ¿Qué es la democracia? D.F.:: Taurus.

Torgerson, C. (2003). Systematic reviews, Cornwall: Continuum. 